\title{
Molecular Mechanisms of Pesticide Toxicity in Humans
}

\section{Jamil K*}

Bhagwan Mahavir Medical Research Centre, India

*Corresponding author: Kaiser Jamil, Head-Department of Genetics, Bhagwan

Mahavir Medical Research Centre, 10-1-1, Mahavir Marg, India, Email: Kaiser.jamil@gmail.com

\section{Mini Review \\ Volume 4 Issue 4}

Received Date: October 01, 2019

Published Date: November 26, 2019

DOI: $10.23880 /$ act- 16000171

\section{Abstract}

Pesticides are chemicals designed to kill a variety of pests, such as insects, weeds, rodents and fungi generally to improve crop production. Improper and widespread usage of pesticides and usage of banned pesticides leads to neurological and haematological complications in individuals. This scenario is highly prevalent in India and other developing countries, where there is continuous usage of different pesticides, including banned ones, based on the season and crop produced. The degree of toxicity to individuals depends upon the nature of the pesticides, their environmental concentration and factors such as temperature, humidity, $\mathrm{pH}$, oxygen concentration, etc. It became evident that persistent usage of pesticides had an adverse impact on the ecological communities. Since most of the pesticides are acetylcholine inhibitors and nerve poisons, this has led to a number of extensive epidemiologic investigations exploring the possible impact of their exposures on human health. The mechanism of toxicity is the inhibition of acetlycholenesterase resulting in the accumulation of neurotransmitter acetylcholine and continuous stimulation of acetylcholine receptors. Humans affected by these chemicals suffer from acute, subacute or chronic toxicity followed by neurological sequel, depending not only on the extent and route of exposure but also on the chemical structure of the compound. Aerial sprays or dusts can be rapidly absorbed through skin and mucous membranes or by inhalation causing toxicological problems. Cholinergic toxicity occurs when too much acetylcholine is present in the synapse leading to excessive parasympathetic effects. This review deals with the molecular mechanisms of cyto and genotoxicity of pesticides and their possible implications on human health.

Keywords: Pesticide Toxicity; Acetlycholenesterase; Haematological Complications; Genotoxicity

\section{Introduction}

Organophosphate/ carbamate compounds are widely used by farmers in India and world over for crop protection and are thus in direct contact with humans. Common pesticides used in homes and lawns are now being shown in medical research to accelerate aging of the immune and nervous system resulting in serious health problems years after exposure. Hence in this article an attempt is made to present their molecular mechanisms of toxicity. There are several possible ways in which humans can be exposed to pesticides, and thus the toxic effects of pesticides may have consequences for 
consumers of food, as well as for agricultural farmers, production workers, formulators, and other applicators [1]. The mode of action pesticides at the cell and molecular level are diverse and some of these mechanisms have been detailed in the publications has been demonstrated often cannot be specifically classified $[2,3]$. However, it is well known that many commonly used pesticides in general are extremely neurotoxic since they irreversibly inhibit acetylcholinesterase, an enzyme that hydrolyzes the neurotransmitter acetylcholine at neuromuscular junctions and brain cholinergic synapses. Presently, more than 10000 chemicals are classified as pesticides. Exposure of humans to a multitude of chemicals, and the presence of pesticides in the environment has been implicated with an increased cancer risk [4]. Biomonitoring studies suggest a genotoxic hazard at the group level. Exposure to pesticides has been associated with increased risks of certain cancers, adverse reproductive outcomes, and neurotoxicity among farmers and other pesticide applicators.

Exposure to pesticides (neurotoxic substances) can produce immediate effects (acute) and/or long-term effects (chronic). In both cases, the effects can be reversible and disappear over time following reduction or cessation of exposure, or result in permanent, irreversible damage. The severity of acute and chronic nervous system impairment depends on exposure dose, which includes both the quantity and duration of exposure. Like alcohol and recreational drugs, many neurotoxic substances may initially be excitatory, producing a sensation of well-being or euphoria and/or speeding up motor functions; as the dose increases in quantity or in time, these same neurotoxins will depress the nervous system. Indeed, nercosis (a state of stupor or insensibility) is induced by many neurotoxic substances, which are mind-altering and depress the central nervous system [5]. Time and again evidences have suggested, OPs have direct stimulatory effects on cholinergic receptors at extremely low concentrations. Pesticides can be tested across many end points, either pharmacologically or toxicologically. The pattern of activity across these endpoints should encode information about the biological nature of the tested chemical [6].

Certain tests, such as DNA adduct formation, are used for measuring biologically effective dose, whereas others are considered to measure early effects, such as chromosomal aberrations. The measurement of acetylcholinesterase level can be used as a biomarker to evaluate the exposure to organophosphates and carbamate pesticides. Organophosphates and carbamates are anti-esterase insecticides, and exert their acute effects by causing overstimulation at cholinergic nerve terminals [7]. This process occurs in both insects and humans.

Normally, acetylcholinesterase catalyzes the degradation of the neurotransmitter acetylcholine in the synapse (yellow panel in Figure 1). Acetylcholine (ACh) is a major neurotransmitter, or nerve-signaling chemical, that acts as a signaling chemical both in the brain and elsewhere in the body. Organophosphate pesticides phosphorylate acetylcholine, thereby reducing the ability of the enzyme to break down the neurotransmitter (red panel in Figure 1).

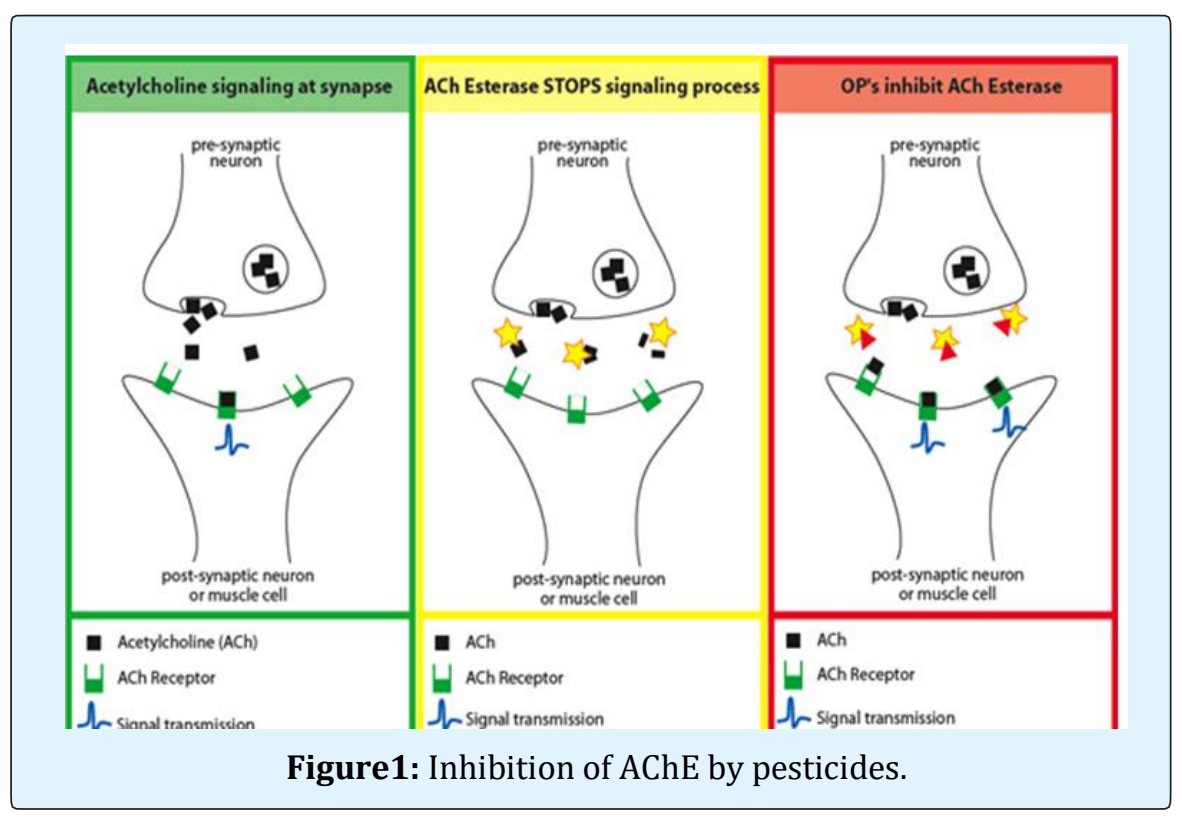

Jamil K. Molecular Mechanisms of Pesticide Toxicity in Humans. Adv Clin Toxicol 2019, 4(4): 000171. 
This produces an accumulation of acetylcholine in the central and peripheral nervous systems, resulting in an acute cholinergic syndrome via continuous neurotransmission [8]. The clinical onset of cholinergic over-stimulation can vary from almost instantaneous to several hours after exposure.

A decrease in a farmer's concentration of acetylcholinesterase indicates over-exposure to such pesticides; the affected worker (often asyrnptomatic) must be removed from exposure, to prevent acute poisoning. Cholinesterase's (Acetyl cholinesterase AChE and butyryl cholinesterase BuChE) are characterized as the main enzymes involved in the toxic effect of these compounds, including molecular forms. Blood cholinesterase decreases proportionally to the degree of acute organophosphate or carbamate intoxication. Organophosphorus and carbamate compounds irreversibly and reversibly inhibit the enzyme acetylcholinesterase (AChE), resulting in excessive accumulation of acetylcholine, leading to the paralysis of cholinergic transmission in the CNS, autonomic ganglia, parasympathetic nerve endings, some sympathetic nerve endings and neuromuscular junction. The inactivation of acetylcholinesterase causes overstimulation of the nervous system, which produces such symptoms as headache, dizziness, nausea, stomachache, and weakness. The types and severity of cholinesterase inhibition symptoms depend on the toxicity of pesticide, the amount of pesticide involved in exposure, the route and duration of exposure. While it is clear that organophosphate and carbamate insecticides pose human health risks through at least one common mode of action, these compounds pose widely different levels of risk per unit of exposure, and also per acre-treated. Depression of nerve or neuromuscular acetylcholinesterase is most indicative of adverse effects, while depression of blood cholinesterase provides a useful indicator of potential impairment. At the same time detection of AChE in the blood gives an indication of the extent of nervous damage caused by the test compounds. In developed countries, erythrocyte Acetylcholinesterase (EC 3.1.1.7) is routinely measured because it is functionally like the neuronal acetylcholinesterase. In addition, $\mathrm{AChE}$ is believed to play non-classical roles in nerve and muscle development and in hematopoiesis.

A great number of in- vitro methods, which have been evaluated, are in the process of validation [9-11]. The implementation of alternative methods in toxicity testing has been established in the European Union through several regulations. A limited number of 'field' studies have also been done aiming at the evaluation of genetic risk, at least at the somatic level, for the human population [12]. An association between occupational exposure to complexes of pesticides and presence of chromosomal aberrations has been established in several cases The fact remains that xenobiotics such as pesticides persist in the environment for a long period of time, along with products of their biotransformation. They may remain and interact with the environment and living organisms in multiple ways, according to their nature and chemical structure, dose and targets. Knowing the impact of pesticides and other related chemicals is very important and of great interest to human health risk assessment since potentially everyone can be exposed to these compounds, which may lower the immune response and cause diseases like malnutrition, atherosclerosis, inflammation, pathogen invasion, nerve injury, and susceptibility to infectious diseases, and metabolic syndrome (through food). Despite the economic and disease control benefits resulting from pesticide use, humans sometimes pay a heavy price in terms of health and environmental problems.

\section{Conflict of Interest}

None

\section{References}

1. Jamil K (2019) Health effects of pharmaceuticals and personal care products. Title of the book: Pharmaceuticals and Personal Care Products: Waste Management and Treatment Technology [Eds.], Prasad MNV, et al. Publisher: Elsevier, pp: 115-128.

2. Das PG, Jamil K (2009) Human serum paraoxonase (PON-1) gene polymorphisms and association with chronic symptoms of pesticide toxicity in Indian farmers. Toxicological \& Environmental Chemistry 91(1): 177-185.

3. Das P, Shaik AP, Jamil K (2007) Cytotoxicity and Genotoxicity induced by the pesticide Acephate on Cultured Human Peripheral Blood Lymphocytes. The Internet Journal of Toxicology 5(2): 1-6.

4. Jamil K (2001) BK\# Bioindicators and Biomarkers of Environmental Pollution and Risk Assessment. Published by: Science Publishers, Inc, Enfield (Nh), USA and Plymouth, UK.

5. Das PG, Shaik AP, Jamil K (2006a) Estimation of apoptosis and necrosis caused by pesticides in vitro 
on human lymphocytes using DNA diffusion assay. Drug Chem Toxicol 29(2): 147-156.

6. Das PG, Jamil K, Rahman MF (2006) Effect of four Organophosphorus Compounds on Human Blood Acetylcholinesterase: In vitro Studies. Toxicol Mech Methods 16(8): 1-5.

7. Das PG, Shaik AP, Jamil K (2006b) Cytotoxicity and Genotoxicity induced by the pesticide Profenofos on cultured human peripheral blood lymphocytes. Drug Chem Toxicol 29(3): 313-322.

8. Naravaneni R, Jamil K (2005) Cytogenetic Biomarkers of Carbofuran Toxicity Utilizing Human Lymphocyte Cultures In-Vitro. Drug Chem Toxicol 28(3): 359-372.

9. Naravaneni R, Jamil K (2007) Determination of Aecetylcholiesterase levels and genotoxic effects in farmers exposed to pesticides. Human and Experimental Toxicology 26(9): 723-731.

10. Jamil K, Shaik AP, Laxmi AJ (2005a) Pesticide Induced Cytogenetic Risk Assessment in Human Lymphocyte Culture In-Vitro. Bull Environ Contam Toxicol 75(1): 7-14.

11. Jamil K, Shaik AP, Mahboob M, Krishna D (2004) Effect of Organophosphorus and Organochlorine Pesticides (Monocrotophos, Chlorpyriphos, Dimethoate and Endosulfan) On Human lymphocytes In-vitro. Drug Chem Toxicol 27(2): 133-144.

12. Das P, Shaik AP, Jamil K (2006c) Estimation of Necrosis caused by pesticides In Vitro on human lymphocytes using DNA Diffusion Assay. Drug and Chemical Toxicology 29(6): 1-10. 